\title{
Based on Petri Nets and Hybrid Genetic-Tabu Search Approach to Scheduling Optimization for Dual-Resource Constrained Job Shop
}

\author{
LIANG $\mathrm{Di}^{1, \mathrm{a}}$, LIU $\mathrm{Si}^{1, \mathrm{~b}}$ and TAO Ze $\mathrm{e}^{2, \mathrm{c}}$ \\ ${ }^{1}$ School of Mechanical Engineering, Shenyang University, Shenyang, P.R.China \\ ${ }^{2}$ School of Mechanical Engineering, Shenyang Science Technology University, Shenyang, \\ P.R.China.

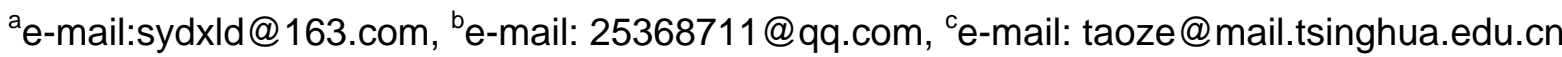

Keywords: petri net; controller; genetic algorithm; tabu search; dual-resource; job shop scheduling; optimization

\begin{abstract}
A Petri net with controller is used to model discrete events in flexible job shop scheduling, the objective of scheduling problems is to minimize make-span, the scheduling results is obtained based on genetic and Tabu Search (TS) algorithm. It is proved capable of providing optimized schedule to the job-shop where the machine tool and manpower resources are both constrained. After crossover and mutation operations, an optimal or suboptimal scheduling plan can be found. The result of the test shows that this method is feasible and efficient.
\end{abstract}

Job shop scheduling is the primary content and critical technology of the production management of CIMS, in the past during 30 years, a lot of researchers and results focus on it, but most of them deal with single objective and resource scheduling problems which are fixed processing route and constrained by machine, and production period is its optimal objective. Nevertheless, the production styles are variable and processing route is determined under the special condition. Moreover, job shop scheduling problem is the most difficult combined constraints optimal problem and typical NP hard problem which can't be solved in polynomial time by an effective algorithm. Up to date, there are some traditional algorithms to solve JSP problem [1-2]. About paper [3], Petri net is used to construct JSP model and L1 which combines branch and bound algorithm with dynamic program is used to search its optimal solution. And paper [4], the job sequence is assigned by genetic code firstly, and then filtered beam search which based on breadth-first algorithm is adopted to search. In recent years, the Petri net as a discrete event dynamic system modeling and analysis tool[5], has been successfully applied to the flexible manufacturing system modeling, analysis and control. Some researchers put forward to be based on Petri network scheduling method, but only on the production line itself can not reflect the modeling, as in the production line of external control scheduling strategy influence.

A hybrid algorithm is proposed to solve dual-resource scheduling problem in flexible production environment, and Petri net model with a controller, which can effectively model control. It combines the advantage of global search ability of GA with the self-adaptive merit of Tabu Search (TS) and improves its convergence. It is proved capable of global and part searching ability and efficient.

\section{Problem Description}

This scheduling model describes different parts have many processing routes in a job shop. A production plan is made to determine the processing route for each part, and it is also required to satisfy the shortest makes-pan.

Scheduling objective is

$\min \mathrm{Z}$

Constraints are:

1)the last process of the jth processing route of part $i$

$T_{i j h m} \leq Z$ 
2)its not last process of the jth processing route of part $i$

$$
\begin{aligned}
& T_{i j h m}-T_{i j(h-1) g} \geq t_{i j h m} \\
& \forall i, j, m, h, g, \quad h \neq 1
\end{aligned}
$$

3)Some processes in the jth processing route of part $i$ and the qth processing route of part $p$ are all need to processed by machine $\mathrm{m}$.

$T_{i j h m}-T_{p q s m}+H Y_{i j h p q s m} \geq t_{i j h m}$

4)Some processes in the jth processing route of part $i$ and the qth processing route of part $p$ are all need to processed by worker $\mathrm{w}$.

$T_{i j h w}-T_{p q s w}+H Y_{i j h p q s w} \geq t_{i j h w}$

$\mathrm{Z}$ is make-span, $\mathrm{i}$ is part, $\mathrm{j}$ is a processing route of a part, $\mathrm{m}$ is a machine, $\mathrm{h}$ is the hth process of the processing route of a part, $t_{i j h m}$ is the processing time which the hth process of the jth processing route of part $\mathrm{i}$ is processed by machine $\mathrm{m}, T_{i j h m}$ is the finish time which the hth process of the jth processing route of part $\mathrm{i}$ is processed by machine $\mathrm{m}, \mathrm{H}$ is a very large positive number, $t_{i j h w}$ is the processing time which the hth process of the jth processing route of part $\mathrm{i}$ is processed by machine $\mathrm{m}, T_{i j h w}$ is the finish time which the hth process of the jth processing route of part $\mathrm{i}$ is processed by worker w, $Y_{\text {ijhpqsm }}$ is the distinguish condition which is process $\mathrm{h}$ and $\mathrm{s}$ of machine $\mathrm{m}$, when process $\mathrm{h}$ and $\mathrm{s}$ are all processed by machine $\mathrm{m}$, if the hth process of the jth processing route of part $\mathrm{i}$ is prior to the sth process of the qth processing route of part $\mathrm{p}$, then $Y_{i j h p q s m}=1$ or $Y_{i j h p q s m}=0, Y_{i j h p q s w}$ is the distinguish condition which the process $h$ or $s$ is processed by worker $w$, when process $h$ and $s$ are all processed by worker $\mathrm{w}$, if the hth process of the jth processing route of part $\mathrm{i}$ is prior to the sth process of the qth processing route of part p, then $Y_{i j h p q s w}=1$, or $Y_{i j h p q s w}=0$.

\section{Scheduling Optimization Based on Hybrid Genetic-Tabu Search Algorithm}

TS is effective to solve combinatorial optimization problems, which searches criteria in neighbor region, move to a feasible efficiently solution [6]-[8]. But it exits a problem which adjusts different parameters in this approach, and parameter selection has directly effect on final result, then hence to a poor Robust. As for GA only adjust several parameters rather than a single solution in the population, therefore GA is a supplement of TS. Fig. 1 shows the hybrid structure of GA and TS. In detail, this approach takes the new individual which produced by crossover and mutation of GA as the neighbor region $V\left(x_{n}\right)$ of present solution $x_{n}$ of TS, then searches each individual of $V\left(x_{n}\right)$.

In this paper, GATS algorithm can choose many solutions which can pass through the restrictions from neighbor region $V\left(x_{n}\right)$, and it denotes an important characteristics which GA is based on population rather than individual. If we take the module TS away in Fig.1, then the whole structure is the traditional GA. Module GA constructs the neighbor region of TS and better initial solution. The idea of the hybrid structure makes the new individual which pass tabu restrictions accepted really. So it can decrease those individual which loss valid gene and its fitness is lower than parents, on the other hand it also supplies more opportunity for the lower fitness individual to join the crossover and mutation, which avoids the premature convergence and to balance the exploration and exploitation abilities of simple GA.

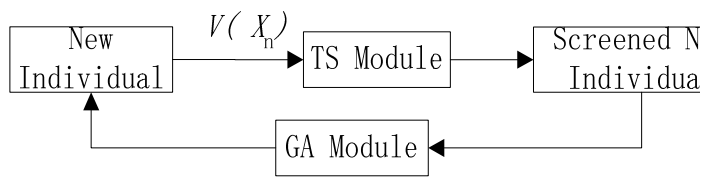

Figure1. Hybrid GA-TS structure of GATS algorithm 


\section{Simulation Experiment of Hybrid Genetic-Tabu Search}

Because the Petri net in the state of the system is expressed in terms of graphic and distributed virtue and the calculation efficiency superiority, many scholars have studied the controlling synthesis problems of the discrete event system modeled by the Petri net[9]. A controller of the Petri net model can not only reflect more than one processing route for machining products of the flexible production line and resource constraints, and the design of controller guarantees that the system satisfies the desired properties, deadlock avoidance, especially for a large scale system, its advantage is more obvious.

To take account of a flexible manufacturing system, 6 machines of 3 type, machine 1 and 2 are type I ; machine 3 is type II ; machine 4, 5 and 6 are type III. 10 jobs are assigned to be processed, each job consists 1 to 4 operations. Worker task parameter and task list are given by Table 1 and Table 2.

TABLE 1 Worker task parameters

\begin{tabular}{|c|c|c|c|c|c|c|}
\hline Worker & Machine 1 & Machine 2 & Machine 3 & $\begin{array}{c}\text { Machine } \\
\mathbf{4}\end{array}$ & Machine 5 & Machine 6 \\
\hline $\mathbf{1}$ & Do & Do & - & - & - & - \\
\hline $\mathbf{2}$ & - & Do & Do & - & - & - \\
\hline $\mathbf{3}$ & - & - & - & Do & Do & \\
\hline $\mathbf{4}$ & - & - & - & - & Do & Do \\
\hline
\end{tabular}

TABLE 2 Example with 3 machines and 7 jobs

\begin{tabular}{|c|c|c|c||c|c|c|c|}
\hline Job & Process & $\begin{array}{c}\text { Processing } \\
\text { Time }\end{array}$ & $\begin{array}{c}\text { Machine } \\
\text { Type }\end{array}$ & Job & Process & $\begin{array}{c}\text { Processing } \\
\text { Time }\end{array}$ & $\begin{array}{c}\text { Machine } \\
\text { Type }\end{array}$ \\
\hline 1 & 1 & 12 & II & 5 & 2 & 10 & III \\
\hline 1 & 2 & 9 & I & 5 & 3 & 10 & I \\
\hline 1 & 3 & 5 & III & 5 & 4 & 8 & III \\
\hline 2 & 1 & 6 & II & 6 & 1 & 6 & II \\
\hline 2 & 2 & 6 & III & 7 & 1 & 6 & III \\
\hline 2 & 3 & 8 & I & 7 & 2 & 8 & III \\
\hline 3 & 1 & 5 & I & 8 & 1 & 12 & I \\
\hline 3 & 2 & 9 & II & 8 & 2 & 8 & II \\
\hline 4 & 1 & 4 & I & 8 & 3 & 8 & III \\
\hline 4 & 2 & 6 & III & 9 & 1 & 5 & I \\
\hline 4 & 3 & 10 & I & 9 & 2 & 8 & III \\
\hline 5 & 1 & 5 & I & 10 & 1 & 7 & III \\
\hline
\end{tabular}

Construct the PN model of part 1, shown as Fig.1, and these symbols mean: $\mathrm{P}_{\mathrm{i}}$ is the start state of the ith part, $P_{i}^{m}$ is the jth machine, $P_{i, j, k}$ means the jth process of the ith part is machined by the kth machine, $t_{i, j, k}^{s}$ means the start of $P_{i, j, k}, t_{i, j, k}^{e}$ means the end of $P_{i, j, k}, P_{i, j}^{b}$ means the buffer zone which the jth process of part $\mathrm{i}$ is finished, $P_{1}^{f}$ means the ith part is finished. The dashed line portion is the controller; two kinds of behaviors are mainly controlled by it:

1) The first buffer zone can not overflow, neither nor underflow. Machine 3 can not be operated

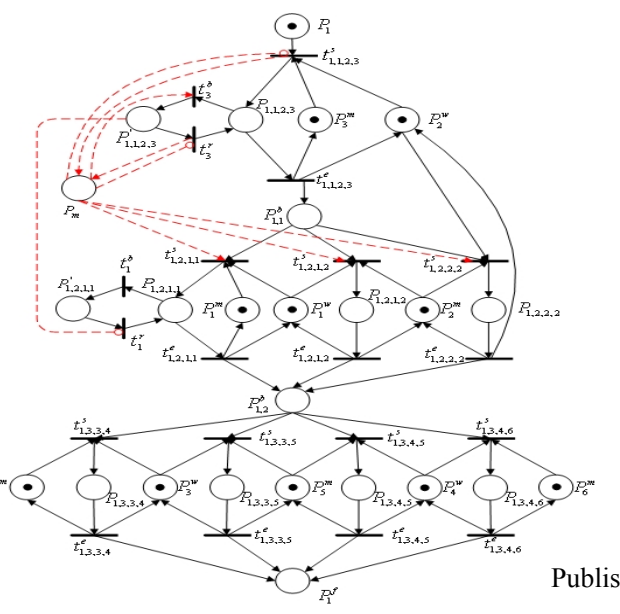

Figure 2 Petri net model of part 1 when parts exist in buffer zone, that is $m\left(P_{1,1,2,3}\right)+m\left(P_{1,1}^{b}\right) \leq 1$;

2) Machine 3 has the repair and return to normal work (busy) priority than machine 1 .

According to this method, the Petri net model of the other parts are constructed in turn, and these models are connected together by resources represented machines, then the whole model can be obtained. Due to the huge graphic, this is omitted here. Presented Petri net with controller in addition to the traditional Petri net modeling ability, also for system maintenance, all kinds of priority 
characteristics are described, for production system is more perfect and more accurate characterization.

The simulated example is described in Table1 and Table2. Population size is 50, crossover probability is 0.8 , and mutation probability is 0.1 . The scheduling result is given in Fig. 3 , after 50 evolutionary generations, the grey color denotes that machine or worker is busy. Objective function value is 55. If the optimal solution is not only one, this approach can also search them all. Fig. 3 and 4 only show one optimal solution.Fig. 3 describes the assignments of processing and machine. The horizon coordinate shows processing time, and vertical coordinate shows machine, four symbols indicate different job and processing in Gannt picture, the former two symbols are job number, the third is process number, the last one is worker; for example, "0511" on the machine 1 means worker 1 processed job 5's first process, “0122” on the machine 2 means worker 2 processed job 1's second process. It is easy obtained to distinguish job’s process by time relation of horizon coordinate, and we can also find start time and finish time of any processing in the picture.

Fig.4 describes the corresponding relationship among job, worker and machine, the horizon coordinate shows processing time, and vertical coordinate shows worker, four symbols indicate a process, the former two are job number, the third is processing number, the last one is machine number; for example, for worker 3, “1014” means job 10's first process is processed on the machine 4 , and we can find processing time on the horizon coordinate. It is easy obtained the job's processing time and worker's workload, and we can also find start time and finish time of any processing in the picture. e.g., the workload of worker 1 is $54(5+12+4+5+10+10+8)$, the workload of worker 2, 3 and 4 are 54, 34 and 38 respectively.

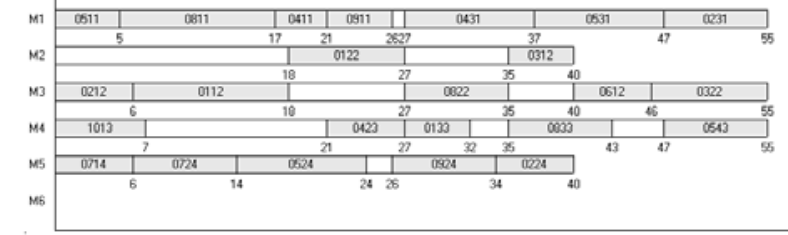

Figure 3. Assignments of machines as constrained by dual-resource

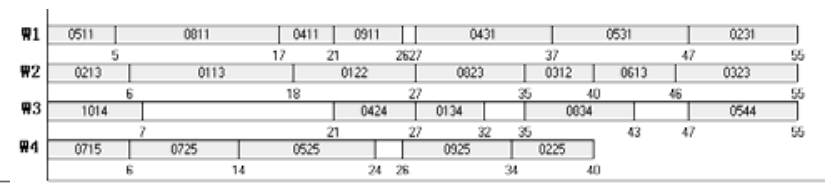

Figure 4. Assignments of workers as constrained by dual-resource

\section{Conclusions}

We propose in this paper the hybrid GATS approach for the dual-resource job shop scheduling problem in the system of workshop control flow on the basis of Petri net with controller. The method can not only reflect many processing routes for machining products processing and resource constraints of the flexible production line, but also on the system equipment maintenance, all kinds of priority characteristics are described, for production system is more perfect and more accurate description. The controller design and system operation to meet the desired performance, avoid deadlock, especially for a large scale system, its advantage is more obvious. The chosen objective is production efficiency. The simulation experiment shows that this method is capable of high searching precision.

\section{References}

[1]M. R. Garey, D. S. Johnson, R. Sethi, “The complexity of flow shop and job shop scheduling,” Mathematics of Operations Research, vol. 261, pp.117-129, Jan. 1996.

[2]J. Blazewicz, K. H. Ecker, Scheduling in Computer and Manufacturing Systems. 2nd Rev Ed. Berlin, Springer-Verlag, 1996, pp.5-30.

[3]D. Y. Lee, F. Dicesare, "Scheduling flexible manufacturing systems using Petri net and heuristic search," IEEE Transaction on Robotics and Automation, vol.10, pp.123-132, Feb. 1994.

[4]W. Holsapple, V. S. Jacob, R. Pakath, J. S. Zaveri, “A genetics-based hybrid scheduler for generating static schedules in flexible manufacturing contexts,” IEEE Transaction on Systems, 
Man and Cybernetics, vol. 23, pp.953-972, Apr. 1993.

[5]CHEN Jyh-horng, FU Li-chen, LIN Ming-hung,etal. Petri-net and GA-based approach to modeling, schedul-ing, and performance evaluation for wafer fabrication[J]. IEEE Transaction on Robotics and Automation, 2001,17(5):619-636.

[6]F. Glover, J. P. Kell, M. Laguna, "Genetic algorithm and tabu search: hybrids for optimizations, "Computers and Operations Research, vol. 22, pp.111-134, Jan.1995.

[7]Laguna M, Barnes J W, Glover F. "Tabu search methods for a single machine scheduling proplem, Journal of Intelligent Manufacturing, ”vol. 56, pp.63-74, Feb. 1991.

[8] M. Laguna, J. W. Barnes, F. Glover, "Intelligent scheduling with tabu search: an application to jobs with linear delay penalties and sequence-dependent setup costs and times," Journal of Applied Intelligence, vol. 86, pp.159-172, March 1993.

[9] M.Uzam. Synthesis feedback control elements for discrete event systems using Petri net models and theory of regions. The International Journal of Advanced Manufacturing Technology, 2004, 24: 48-69. 